\title{
A STRATEGIC MODEL FOR DYNAMIC TRAFFIC ASSIGNMENT*
}

\author{
by \\ Younes Hamdouch ${ }^{1}$ \\ Patrice Marcotte ${ }^{1}$ \\ Sang Nguyen ${ }^{1}$
}

23 octobre 2002

*This research was supported in part by the Natural Sciences and Engineering Research Council of Canada (NSERC) and by the Fonds pour la formation de chercheurs et l'aide à la recherche (FCAR).

${ }^{1}$ Département d'informatique et de recherche opérationnelle et Centre de recherche sur les transports, Université de Montréal, C.P. 6128, succursale Centre-ville, Montréal, CANADA H3C 3J7 


\begin{abstract}
We propose a model of dynamic traffic assignment where strategic choices are an integral part of user behaviour. The model provides a discrete-time description of flow variation through a road network involving arcs with rigid capacities. A driver's strategy assigns, to each node of the network, a set of arcs in the forward star of that node, sorted according to a preference order. The main element of the model is a 'within-day' submodel where strategic volumes are loaded onto the network in accordance with the first-in first-out discipline and user preferences. An equilibrium assignment is achieved when expected delays are minimal, for every origin-destination pair. We prove the existence of such an assignment and provide numerical results on test networks.
\end{abstract}

Keywords: dynamic traffic assignment, strategy, hyperpath, capacities.

\title{
RÉSUMÉ
}

Cet article propose un modèle dynamique discret pour le trafic routier où le comportement des usagers est dicté par des stratégies. Le modèle permet d'analyser la variation des flots de véhicules dans les réseaux dont les arcs sont munis de capacités rigides. Chaque stratégie associe à tout nœud du réseau un ensemble d'arcs incidents, ordonné en ordre de préférence décroissante. L'élément central du modèle est un sous-modèle où les volumes stratégiques sont chargés sur le réseau en respectant les priorités temporelles (premier arrivé premier servi) ainsi que les préférences. Un équilibre est atteint lorsque tous les automobilistes sont affectés à des plus courts chemins reliant leur origine et leur destination. Nous démontrons l'existence d'une affectation respectant ce principe d'équilibre et présentons des résultats numériques sur des réseaux test.

Mots-Clés: trafic routier, affectation d'équilibre dynamique, stratégie, hyperchemin, capacités. 


\section{Introduction}

Traffic assignment has been the topic of many studies in the last four decades, and it can be safely argued that the static case is settled by now. However, extending the results from a static to a dynamic environment is far from trivial and, as no agreement on an ideal model has been reached within the scientific community, dynamic traffic assignment remains a topic of active research.

Macroscopic dynamic models belong to one of three classes: models using exit functions (Merchant and Nemhauser [10], Carey [2], Drissi-Kaïtouni and Gendreau [4]), models based on space-time networks (Drissi and Hamada-Benchekroun [3], Zawack and Thompson [15]) and models using arc travel delays (Friesz and al. [5], Astarita [1]). In all these models, users travel along paths of the underlying network, which path cannot be modified en route. If congestion levels become very high, however, users may choose to switch path rather than experience unduly delays on a given route. Our proposal for dealing with this situation is to assume that users behave strategically and modify their route choice according to congestion conditions. More precisely, a strategy associates with each node of the network an ordered set of successor nodes, whose access is controlled by three factors: the arrival time at the node, the capacity of the corresponding arc, the number of users with overlapping strategies.

This model adapts the strategic approach of Marcotte, Nguyen and Schoeb [9] and Hamdouch [6] to a time-varying environment. As in most dynamic models, the main challenge consists in performing efficiently the 'loading' operation, whereby strategic volumes are converted into path and arc volumes. It is a key feature of our approach that the loading preserves the order of arrival of users at the nodes of the network, and thus automatically fulfills the FIFO (First-In First Out) rule.

The remainder of this paper is organized as follows. In the next section, we introduce the strategic concepts underlying the dynamic assignment model and formulate the equilibrium problem as a variational inequality. Based on these results, we develop an algorithmic framework and provide numerical results. Throughout the paper, we assume that time-dependent demand and departure times are known, and that congestion is the sole consequence of waiting time at the tails of congested nodes.

\section{A strategic dynamic assignment model}

Let $G=(N, A)$ be a network with node set $N$, arc set $A$ and time-varying demand $d_{q r}^{t}\left(t=0,1, \ldots, T^{\prime} \leq T\right)$, for users departing origin node $q$ at $t$ and bound for destination node $r$. The objective of our research is to determine strategic volumes and travel times over a fixed time interval $T$ whose expected delays are consistent with the travel costs $c_{a}$ and the arc capacities $u_{a}$. We assume that the time interval is sufficiently large to allow all vehicles to reach their destination by $T$ and that, whenever the number of drivers that wish to access an arc exceeds its capacity, a proportion of those commuters will either travel on an alternative (unsaturated) arc, or wait until an arc become available later in time. All operations take place on a 
time-space network $R=(V, E)$ which is constructed as follows:

- each node $i \in N$ is expanded into $T+1$ nodes $i_{t}, t=0,1, \ldots, T$ :

$$
V=\left\{i_{t}: i \in N, 0 \leq t \leq T\right\}
$$

- each link $a \in A$ is expanded to $T+1-c_{a} \operatorname{links}\left(i_{t}, j_{t+c_{a}}\right)$ such that $t+c_{a} \leq T$; we refer to these links as travel links. The waiting time at the nodes is represented by means of waiting arcs of infinite capacity denoted $\left(i_{t}, i_{t+1}\right)$ where $t=0,1, \ldots, T-1$.

The set of arcs is partitioned into the set $E_{1}$ of access arcs and its complement $E_{2}$, the set of waiting arcs:

$$
\begin{aligned}
E_{1} & =\left\{\left(i_{t}, j_{t+c_{i j}}\right):(i, j) \in A, 0 \leq t \leq T-c_{i j}\right\} \\
E_{2} & =\left\{\left(i_{t}, i_{t+1}\right): i \in N, 0 \leq t \leq T-1\right\} \\
E & =E_{1} \cup E_{2}
\end{aligned}
$$

As an example, the time-space network $R$ of 5 periods corresponding to the example of Figure 1 is illustrated in Figure 2. Note that $R$ is a standard acyclic graph with $|V|=n(T+1)$ and $|E| \leq(m+n) T$ where $n=|N|$ and $m=|A|$.

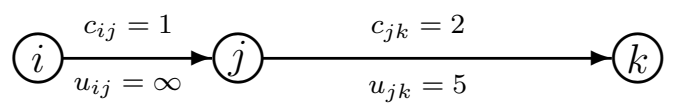

Figure 1: A simple network

\subsection{Strategies}

A strategy $s$ is associated with an origin-destination pair $(q, r)(t)$ and maps every node $j_{t}$ of the time-space network into an ordered set of successor nodes that embodies the arrival instant at $j_{t}$. More precisely, let $E_{j}^{t}$ denote the set of all subsets of the forward star $j_{t}^{+}$associated with node $j_{t}$. A strategy $s$ assigns to each node $j_{t} \in V$ an element $E_{j}^{s t t^{\prime}}\left(t^{\prime} \leq t\right)$ in $E_{j}^{t}$. The set $E_{j}^{s t t^{\prime}}$ is the preference order at node $j_{t}$ for users adopting strategy $s$ and having reached node $j$ at instant $t^{\prime}$. Three strategies for the simple network of Figure 2 are listed below:

preference order: $\quad E_{i}^{s 00} \quad E_{i}^{s 11} \quad E_{i}^{s 22} \quad E_{j}^{s 11} \quad E_{j}^{s 21} \quad E_{j}^{s 22} \quad E_{j}^{s 33} \quad E_{k}^{s 55}$

$\begin{array}{ccccccccc}s_{1}: & {\left[j_{1}\right]} & {[]} & {[]} & {\left[k_{3}, j_{2}\right]} & {\left[k_{4}\right]} & {\left[k_{4}, j_{3}\right]} & {[]} & {[]} \\ s_{2}: & {[]} & {\left[j_{2}\right]} & {[]} & {[]} & {\left[k_{4}, j_{3}\right]} & {\left[k_{4}\right]} & {\left[k_{5}\right]} & {[]} \\ s_{3}: & {[]} & {[]} & {\left[j_{3}\right]} & {[]} & {[]} & {[]} & {\left[k_{5}\right]} & {[]}\end{array}$




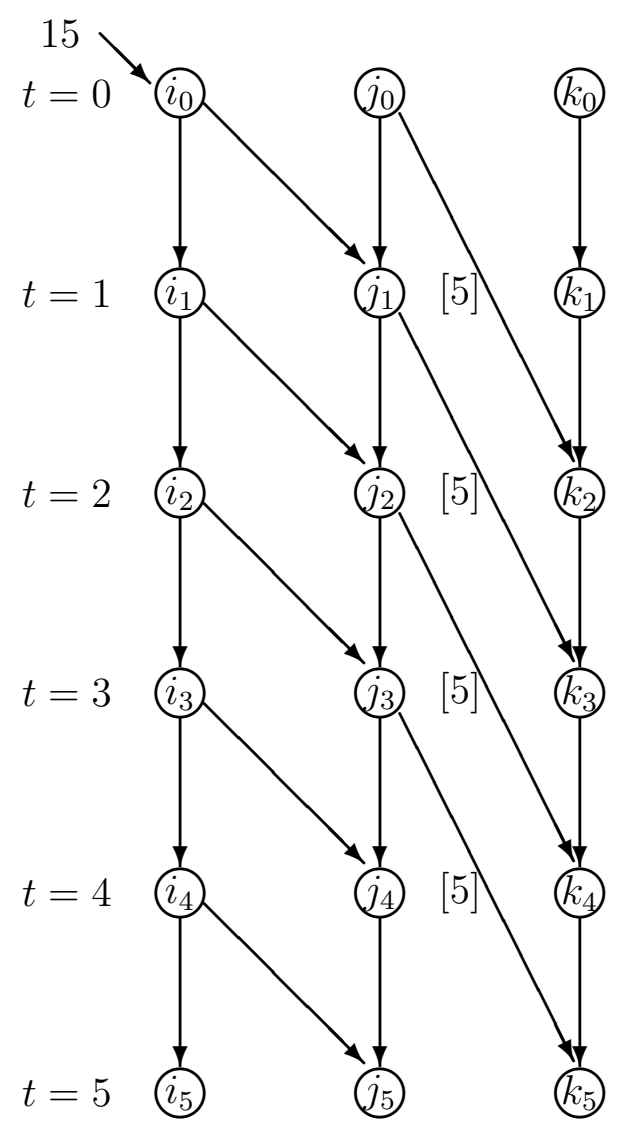

Figure 2: Expansion of the simple network

With any given strategy $s$, we associate the strategic graph $H(s)=\left(V^{s}, E^{s}\right)$, where $E^{s}$ is the union of the set $E_{1}^{s}$ of travel $\operatorname{arcs}\left\{\left(j_{t}, k_{t+c_{j k}}\right) \in E_{1} \quad k_{t+c_{j k}} \in E_{j}^{s t t^{\prime}}, t^{\prime} \leq\right.$ $t\}$ and the set $E_{2}^{s}$ of waiting $\operatorname{arcs}\left\{\left(j_{t}, j_{t+1}\right) \in E_{2} \quad j_{t+1} \in E_{j}^{s t t^{\prime}}, t^{\prime} \leq t\right\}$. Every node $j_{t} \in V^{s}$ is connected to the destination $r(s)$. The strategic graph induced by strategy $s_{1}$ is shown in Figure 3.

The set of all strategies associated with OD pair $(q, r)(t)$ is denoted by $S_{q r}^{t}$ and we denote by

$$
S=\bigcup_{0 \leq t \leq T} S^{t}=\bigcup_{0 \leq t \leq T} \bigcup_{(q, r, t)} S_{q r}^{t}
$$

the set of all strategies.

\subsection{Strategic volumes and strategic costs}

Let $x_{s}^{t}$ represent the number of drivers using strategy $s$ and leaving their origin $q(s)$ at instant $t$. We denote by $x=\left(x_{s}^{t}\right)_{s \in S}$ the vector of strategic volumes and by $X$ the set of all demand-feasible such vectors, i.e.,:

$$
X=\left\{x: \sum_{s \in S_{q r}^{t}} x_{s}^{t}=d_{q r}^{t} \quad \forall(q, r), \forall t \in T\right\}
$$




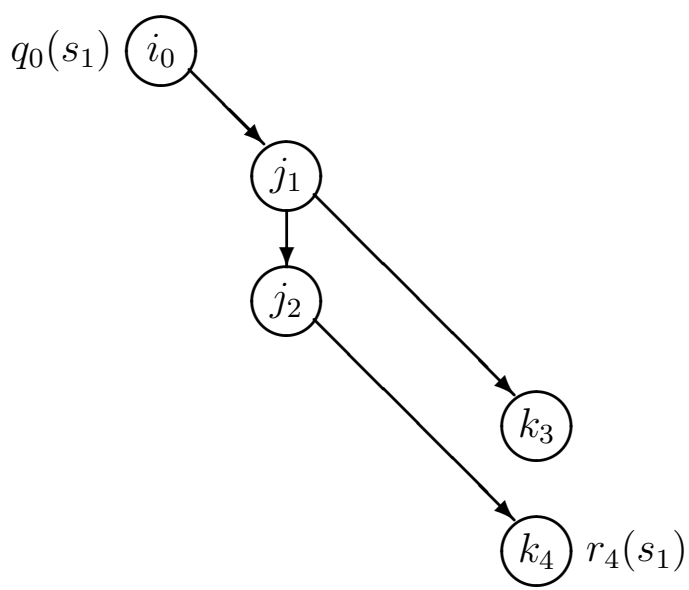

Figure 3: The strategic graph of $s_{1}$ on simple network

The cost of strategy $s$ depends directly on the arc access probabilities $\pi_{j k}^{s t^{\prime}}(x)$ and $\pi_{j}^{s t^{\prime}}(x)$, the latter corresponding to a waiting arc; $\pi_{j k}^{s t^{\prime}}(x)$ (respectively $\pi_{j}^{s t^{\prime}}(x)$ ) is the probability that drivers adopting strategy $s$ access node $k_{t^{\prime}+c_{j k}}$ (respectively $j_{t^{\prime}+1}$ ) from node $j_{t^{\prime}}$. These access probabilities are computed by a dynamic loading process that will be the focus of Section 3. The arc access probabilities induce node access probabilities, computed recursively as follows:

$$
\tau_{j}^{s t^{\prime}}(x)= \begin{cases}0 & \text { if } j_{t^{\prime}} \notin V^{s} \\ 1 & \text { if } j_{t^{\prime}}=q_{t}(s) \\ \tau_{j}^{s\left(t^{\prime}-1\right)} \pi_{j}^{s\left(t^{\prime}-1\right)}+\sum_{k_{t_{c}} \in j_{t^{\prime}}^{-}} \tau_{k}^{s t_{c}} \pi_{k j}^{s t_{c}} & \text { if } j_{t^{\prime}} \in V^{s} \backslash\left\{q_{t}(s)\right\}\end{cases}
$$

where $t_{c}=t^{\prime}-c_{k j}$ is the instant when a user of strategy $s$ leaves node $k$ through arc $(k, j)$.

In a natural fashion, we define the strategic cost of a strategy $s$ with departure time $t$ and destination $q(s)$ as the weighted sum:

$$
C_{s}^{t}(x)=\sum_{t^{\prime} \geq t}\left\{\sum_{\left(j_{t^{\prime}}, k_{t^{\prime}+c_{j k}}\right) \in E_{1}^{s}} \tau_{j}^{s t^{\prime}}(x) \pi_{j k}^{s t^{\prime}}(x) c_{j k}+\sum_{\left(j_{t^{\prime}}, j_{t^{\prime}+1}\right) \in E_{2}^{s}} \tau_{j}^{s t^{\prime}}(x) \pi_{j}^{s t^{\prime}}(x)\right\} .
$$

An equilibrium strategic flow $x^{*}$ is then readily characterized as a solution of the variational inequality

$$
\left\langle C\left(x^{*}\right), x^{*}-x\right\rangle \leq 0 \quad \forall x \in X .
$$

If the cost function $C$ were continuous, the existence of an equilibrium solution would follow directly from classical fixed point theorems, such as Brouwer's or Kakutani's. A technical difficulty arises in the degenerate situation where a null strategic volume wants to access an arc with null residual capacity. This can be resolved exactly as in Hamdouch [7], i.e., by showing that defining the access probability to zero in the degenerate case yields a lower semi-continuous cost mapping $C$. The proof will not be repeated here. 


\subsection{The FIFO rule and flow priority}

A main feature of our model is that the FIFO rule is strictly enforced. At a a node $j_{t^{\prime}} \in V$, this is achieved dividing each group of users $z_{j}^{s t^{\prime}}$ into subgroups according to their arrival instant at node $j$. We denote by $z_{j}^{s t^{\prime \prime} t^{\prime \prime}}$ the strategic volume at node $j_{t^{\prime}}$ adopting strategy $s$ and having reached node $j$ at instant $t^{\prime \prime} \leq t^{\prime}$ and regroup all active strategies into a class $\bar{S}^{t^{\prime} t^{\prime \prime}}$ restricted to users having reached node $j$ at instant $t^{\prime \prime}:$

$$
\bar{S}^{t^{\prime} t^{\prime \prime}}=\left\{s \in S: E_{j}^{s t^{\prime} t^{\prime \prime}} \neq \emptyset, z_{j}^{s t^{\prime} t^{\prime \prime}}>0\right\} .
$$

Based on the access probabilities $\pi_{j k}^{s t^{\prime}}(x)$ and $\pi_{j}^{s t^{\prime}}(x)$, the volume $z_{j}^{s t^{\prime} t^{\prime \prime}}$ is then computed according to the recursion

$$
z_{j}^{s t^{\prime} t^{\prime \prime}}= \begin{cases}0 & \text { if } j_{t^{\prime}} \notin V^{s} \\ x_{s}^{t} & \text { if } j_{t^{\prime}}=q_{t}(s)\left(t^{\prime \prime}=t^{\prime}\right) \\ \pi_{j}^{s\left(t^{\prime}-1\right)} z_{j}^{s\left(t^{\prime}-1\right) t^{\prime \prime}} & \text { if } j_{t^{\prime}} \in V^{s} \backslash\left\{q_{t}(s)\right\} \text { and } t^{\prime \prime} \leq t^{\prime}-1 \\ \sum_{k_{t_{c}} \in j_{t^{\prime}}} \pi_{k j}^{s t_{c}} z_{k}^{s t_{c}} & \text { if } j_{t^{\prime}} \in V^{s} \backslash\left\{q_{t}(s)\right\} \text { and } t^{\prime \prime}=t^{\prime}\left(t_{c}=\left(t^{\prime}-c_{j k}\right) .\right.\end{cases}
$$

In order to control access priorities, we assign the highest priority to users of the first class $\bar{S}^{t^{\prime} 0}$. The strategic volume of the remaining classes are then loaded in increasing order according to their arrival instant at node $j$. This ordering is an integral part of the loading process described in the next section.

\section{The dynamic loading process}

In static models, the arc-path incidence matrix is flow independent. As a consequence, link information can be readily recovered from path flow information. In dynamic models, the situation is different and calls for a mechanism that maps path (or strategic) flows into link flows. In our model, this operation is performed by a loading procedure that assigns users to their preferred available arc, until their first choices becomes saturated. Saturated arcs are then removed from the preference sets and the procedure is iterated until all users are assigned to an outgoing arc.

This procedure will be explained in detail on the example corresponding to figure 4 , where capacities of the physical $\operatorname{arcs}(j, k)$ and $(j, l)$ are set to 10 and 15 , respectively. We focus on the loading process at node $j_{4}$, i.e., the physical node $j$ at instant 4 . A total of 20 users are already in the waiting queue at $j$; among these, 15 users have been in the queue for two periods, and the remaining 5 for one period. They are joined in the queue by 20 users that access node $j$ from node $i$. All users adopt one of either two strategies: $s_{1}$ or $s_{2}$, which are specified by the preference sets $E_{j}^{\text {stt }}$ displayed in the figured. Note that the preference set of a strategy can be time dependent, which it is in our example. Next to each arc is shown its volume, together with the repartition among the two classes.

We initiate the process by loading the strategic flow associated with class $\bar{S}^{42}$, i.e., flow that arrived at node $j$ at instant 2. Since capacity is not exceeded, 3 users from 


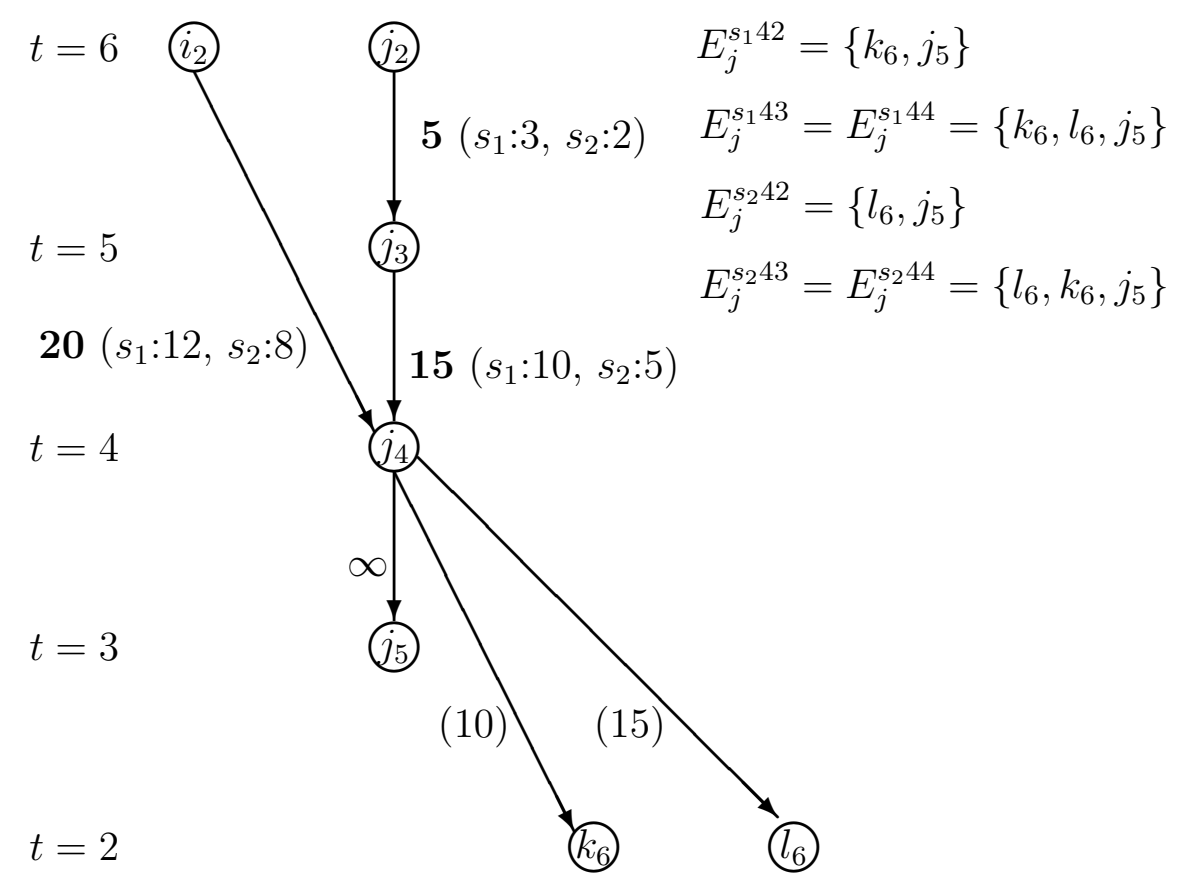

Figure 4: An example of strategic dynamic loading

$s_{1}$ and 2 from $s_{2}$ head for nodes $k_{6}$ and $l_{6}$, respectively. Next, residual capacities are updated and the loading ends for drivers of class. At the second iteration, strategic volumes $z_{j}^{s_{1} 43}$ and $z_{j}^{s_{2} 43}$ of the second class $\bar{S}^{43}$ are assigned to their respective preferred node at a uniform rate. Since the ratio $7 / 10$ is less than $13 / 5, \operatorname{arc}\left(j_{4}, k_{6}\right)$ gets saturated first. At that instant, $\frac{7}{10}$ of $s_{1}$ and $s_{2}$ flows are assigned, i.e., 7 users from $s_{1}$ reach node $k_{6}$ and 3.5 from $s_{2}$ reach $l_{6}$. At iteration 3 , the saturation of $\left(j_{4}, k_{6}\right)$ forces users of strategy $s_{1}$ to adopt their second best choice $l_{6} ; 4.5$ units of volume ( 3 from $s_{1}$ and 1.5 from $\left.s_{2}\right)$ vie for arc $\left(j_{4}, l_{6}\right)$ whose residual capacity is 9.5 . Since capacity exceeds demand, the loading of class $\bar{S}^{43}$ terminates. At the fourth iteration, 12 units of the strategic volume $z_{j}^{s_{1} 44}$ and 8 of $z_{j}^{s_{2} 44}$ compete for the residual capacity (5) of arc $\left(j_{4}, l_{6}\right)$. According to the ratio 1 to 4,3 units from $s_{1}$ and 2 from $s_{2}$ reach node $l_{6}$. A fifth iteration is required to load the unassigned flow to the waiting arc $\left(j_{4}, j_{5}\right)$. The arc probabilities are then obtained by dividing the amount of strategic volume having accessed an arc by the total corresponding strategic volume. More details are provided in Table 1.

A pseudocode of the algorithm, which extends that presented in Hamdouch et al [7] for static networks with priority rules, is given in the appendix. It provides a formal description of the loading of a strategic flow vector $x=\left(x_{s}^{t}\right)_{s \in W^{t} \subset S^{t}, t \leq T}$ with respect to a subset of strategies $W^{t}$ working set. The working set includes at least one strategy for every OD pair $(q, r)(t)$. 
arc: $\quad\left(j_{4}, k_{6}\right) \quad\left(j_{4}, l_{6}\right) \quad\left(j_{4}, j_{5}\right)$

\begin{tabular}{|c|c|c|c|c|}
\hline iter. 1 & $\begin{array}{l}\text { res. cap. } \\
\text { flow }\end{array}$ & $\begin{array}{c}10 \\
3\left(z_{j}^{s_{1} 42}\right)\end{array}$ & $\begin{array}{c}15 \\
2\left(z_{j}^{s_{2} 42}\right)\end{array}$ & $\begin{array}{c}\infty \\
0\end{array}$ \\
\hline iter. 2 & $\begin{array}{l}\text { res. cap. } \\
\text { flow }\end{array}$ & $\begin{array}{c}7 \\
3\left(z_{j}^{s_{1} 42}\right)+7\left(z_{j}^{s_{1} 43}\right)\end{array}$ & $\begin{array}{c}13 \\
2\left(z_{j}^{s_{2} 42}\right)+3.5\left(z_{j}^{s_{2} 43}\right)\end{array}$ & $\begin{array}{c}\infty \\
0\end{array}$ \\
\hline iter. 3 & $\begin{array}{l}\text { res. cap. } \\
\text { flow }\end{array}$ & $\begin{array}{c}0 \\
3\left(z_{j}^{s_{1} 42}\right)+7\left(z_{j}^{s_{1} 43}\right)\end{array}$ & $\begin{array}{c}9.5 \\
2\left(z_{j}^{s_{2} 42}\right)+3.5\left(z_{j}^{s_{2} 43}\right) \\
+3\left(z_{j}^{s_{1} 43}\right)+1.5\left(z_{j}^{s_{j} 43}\right)\end{array}$ & $\begin{array}{c}\infty \\
0\end{array}$ \\
\hline iter. 4 & $\begin{array}{l}\text { res. cap. } \\
\text { flow }\end{array}$ & $\begin{array}{c}0 \\
3\left(z_{j}^{s_{1} 42}\right)+7\left(z_{j}^{s_{1} 43}\right)\end{array}$ & $\begin{array}{c}5 \\
2\left(z_{j}^{s_{2} 42}\right)+5\left(z_{j}^{s_{2} 43}\right) \\
+3\left(z_{j}^{s_{1} 43}\right)+3\left(z_{j}^{s_{1} 44}\right) \\
+2\left(z_{j}^{s_{2} 44}\right)\end{array}$ & $\begin{array}{c}\infty \\
0\end{array}$ \\
\hline iter. 5 & $\begin{array}{l}\text { res. cap. } \\
\text { flow }\end{array}$ & $\begin{array}{c}0 \\
3\left(z_{j}^{s_{1} 42}\right)+7\left(z_{j}^{s_{1} 43}\right)\end{array}$ & $\begin{array}{c}0 \\
2\left(z_{j}^{s_{2} 42}\right)+5\left(z_{j}^{s_{2} 43}\right)+9\left(z_{j}^{s_{1} 44}\right) \\
+3\left(z_{j}^{s_{1} 43}\right)+3\left(z_{j}^{s_{1} 44}\right)+6\left(z_{j}^{s_{2} 44}\right) \\
+2\left(z_{j}^{s_{2} 44}\right)\end{array}$ & $\infty$ \\
\hline
\end{tabular}

access probabilities $\quad \pi_{j k}^{s_{1} 4}=\frac{10}{25} \quad \pi_{j l}^{s_{1} 4}=\frac{6}{25} \quad \pi_{j}^{s_{1} 4}=\frac{9}{25} \quad \pi_{j l}^{s_{2} 4}=\frac{9}{15} \quad \pi_{j}^{s_{2} 4}=\frac{6}{15}$

Table 1: Dynamic loading process at node $j_{4}$ 


\section{Computation of an optimal strategy}

The algorithms that we implemented are extensions to the dynamic case of algorithms presented in [6]. At each iteration, one moves from the current strategic vector toward another vector that incorporates a 'best' response to current traffic conditions, for each origin-destination pair. More precisely we construct, for every OD pair $(q, r)(t)$ and instant $t$, a strategy $s_{q r t}^{*}(x)$ such that:

$$
C_{s^{*}}^{t}(x)=\min _{s \in S_{q r}^{t}}\left\{C_{s}^{t}(x)\right\}
$$

The construction of the solution $s^{*}$ to the above problem is achieved by an algorithm that plays the role of the shortest path algorithm in standard static traffic assignment. It is based on dynamic programming and uses the information (strategic volumes, access probabilities) generated by the loading procedure. One conceptual difficulty is that access probabilities associated with strategies not yet in the working set are unknown. However, a micro loading procedure can be devised to perform that very operation.

The knowledge of the strategic vector $z$ allows us to start the micro-loading at node $j_{t^{\prime}}$ which must comply with the FIFO rule. At each instant $p\left(t \leq p \leq t^{\prime}\right)$, the optimal preference set $E_{j}^{s^{*} t^{\prime} p}$ is constructed by running the micro-loading over the sets $\bar{S}^{t^{\prime} t}, \bar{S}^{t^{\prime} t+1}, \ldots, \bar{S}^{t^{\prime} p-1}$ and $\bar{S}^{t^{\prime} p} \cup\left\{s^{*}\right\}$ given that the users of the optimal (unknown) strategy $s^{*}$ have reached node $j$ at instant $p$. The dependence of the preference set on the arrival instant at node $j$ is key to enforcing the FIFO condition at node $j_{t^{\prime}}$. The recursive process of Nguyen and Pallottino [11] for computing a shortest hyperpath can then be applied to the space-time network $R$. The expected travel cost from node $j_{t^{\prime}}$ to the destination $r_{T}$, denoted $\omega_{p j}^{s^{*} t^{\prime}}$, is obtained from Bellman's generalized Bellman:

$$
\omega_{p j}^{s^{*} t^{\prime}}= \begin{cases}\infty & \text { if } j \neq r, t=T \\ 0 & \text { if } j=r \\ \sum_{k_{t^{\prime \prime}} \in E_{j}^{s^{*} t^{\prime} p}} \pi_{j k}^{s^{*} t^{\prime}}\left(c_{j k}+\omega_{t^{\prime \prime} k}^{s^{*} t^{\prime \prime}}\right)+\pi_{j}^{s^{*} t^{\prime}}\left(1+\omega_{p j}^{s^{*} t^{\prime}+1}\right) & \text { if } j \neq r, t<T\end{cases}
$$

where $t^{\prime \prime}=t^{\prime}+c_{j k}$ is the arrival instant at node $k \in j^{-}$, and where the optimal preference order $E_{j}^{s^{*} t^{\prime} p}$ is constructed efficiently by sorting the labels $c_{j k}+\omega_{k}^{s^{*} t^{\prime \prime}}$ in increasing order, with

$$
\omega_{k}^{s^{*} t^{\prime \prime}}= \begin{cases}\omega_{p k}^{s^{*} t^{\prime \prime}} & \text { if } k_{t^{\prime \prime}}=k_{t^{\prime}+c_{j k}}=j_{t^{\prime}+1} \\ \omega_{t^{\prime \prime} k}^{s^{*} t^{\prime \prime}} & \text { otherwise. }\end{cases}
$$

An optimal solution $s^{*}$, together with its cost vector $\omega^{*}$, is then obtained by solving Bellman's equations in reverse topological order, i.e., starting at instant $T$.

We close this section with an illustration of one iteration of the algorithm within the space-time network. Let us consider the situation depicted in Figure 5, where the relevant data comprises the arrival instants, the arc flows, their associated strategies, the preference orders associated with strategies $s_{1}$ and $s_{2}$, delays, capacities (in 


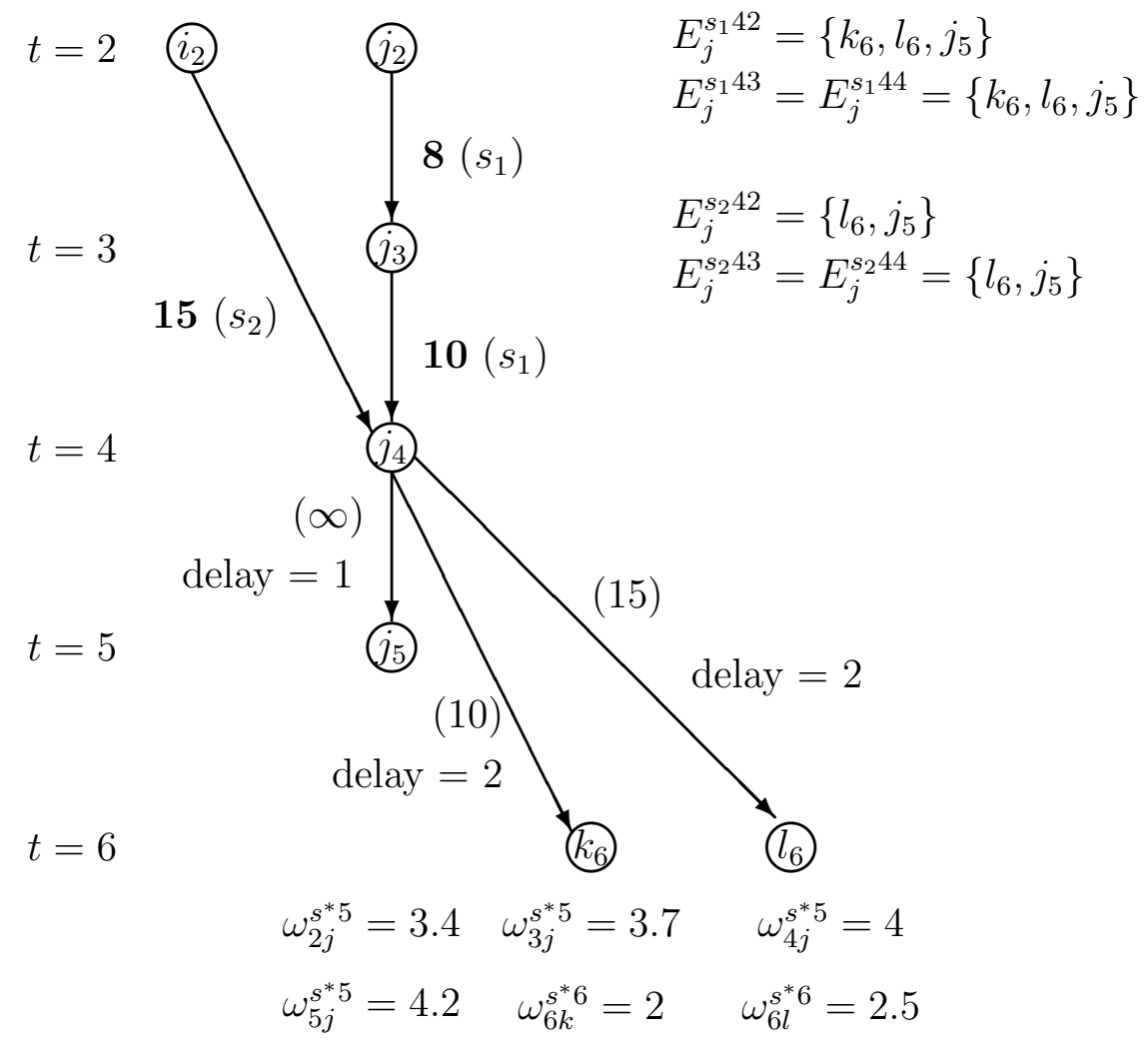

Figure 5: Computing an optimal strategy at node $j_{4}$

parenthesis) and cost-to-go (bottom of picture) that are available at nodes $j_{5}, k_{6}$ and $l_{6}$, since their topological index is higher than that of node $j$. We proceed to compute the best strategic response, starting at the physical node $j$.

The optimal preference orders are obtained by minimizing the sum of arc delays and the corresponding cost-to-go. Accordingly, the inequalities

$$
c_{j k}+\omega_{6 k}^{s^{*} 6}=2+2<1+\omega_{2 j}^{s^{*} 5}=1+3.4<c_{j l}+\omega_{6 l}^{s^{*} 6}=2+2.5
$$

yield $E_{j}^{s^{*} 42}=\left[k_{6}, j_{5}, l_{6}\right]$. In a similar fashion, we derive $E_{j}^{s^{*} 43}=\left[k_{6}, l_{6}, j_{5}\right]$ from

$$
c_{j k}+\omega_{6 k}^{s^{*} 6}=4<c_{j l}+\omega_{6 l}^{s^{*} 6}=4.5<1+\omega_{3 j}^{s^{*} 5}=1+3.7
$$

and $E_{j}^{s^{*} 44}=\left[k_{6}, l_{6}, j_{5}\right]$ from the inequalities

$$
4<4.5<1+\omega_{4 j}^{s^{*} 5}=1+4 .
$$

The algorithm must now determine the values of $\omega_{2 j}^{s^{*} 4}, \omega_{3 j}^{s^{*} 4}$ and $\omega_{4 j}^{s^{*} 4}$ at node $j_{4}$. We start with instant 2 and the strategy set $\bar{S}^{42} \cup\left\{s^{*}\right\}=\left\{s_{1}, s^{*}\right\}$. Since flow does not exceed available capacity, all users from $s_{1}$ and $s^{*}$ head for node $k_{6}$. This yields the access probabilities $\pi_{j k}^{s^{*} 4}=1, \pi_{j}^{s^{*} 4}=\pi_{j l}^{s^{*} 4}=0$ and the cost

$$
\begin{aligned}
\omega_{2 j}^{s^{*} 4} & =\pi_{j k}^{s^{*} 4}\left(2+\omega_{6 k}^{s^{*} 6}\right)+\pi_{j}^{s^{*} 4}\left(1+\omega_{2 j}^{s^{*} 6}\right)+\pi_{j l}^{s^{*} 4}\left(2+\omega_{6 l}^{s^{*} 6}\right) \\
& =1(4)+0(4.4)+0(4.5)=4 .
\end{aligned}
$$


We now repeat the loading operation for users that arrived at instant 3 . We first perform the assignment of the 8 users that arrived at instant 2 to their preferred node $k_{6}$. Next, the residual capacity of arc $(j, k)$ is updated (it becomes $10-8=2$ ) and the loading ends for drivers of strategies $\bar{S}^{42}$. Next, the strategic volumes $z_{j}^{s_{1} 43}$ and $z_{j}^{s^{*}}$ associated with the strategies in $\bar{S}^{43}$ are assigned to their respective preferred node at the uniform rate $2 / 10$. At that instant, $\frac{1}{5}$ of $s_{1}$ and $s^{*}$ flows have reached node $k_{6}$, and the arc $\left(j_{4}, k_{6}\right)$ is saturated. This forces users of strategy $s_{1}$ and $s^{*}$ to adopt their second best choice $l_{6} ; 8$ units of volume from $s_{1}$ and $s^{*}$ access arc $\left(j_{4}, l_{6}\right)$, whose residual capacity (15) exceeds demand (8). This yields the access probabilities $\pi_{j k}^{s^{*} 4}=1 / 5, \pi_{j l}^{s^{*} 4}=4 / 5$ and $\pi_{j}^{s^{*} 4}=0$, from which we derive the cost-to-go

$$
\begin{aligned}
\omega_{3 j}^{s^{*} 4} & =\pi_{j k}^{s^{*} 4}\left(2+\omega_{6 k}^{s^{*} 6}\right)++\pi_{j l}^{s^{*} 4}\left(2+\omega_{6 l}^{s^{*} 6}\right)+\pi_{j}^{s^{*} 4}\left(1+\omega_{3 j}^{s^{*} 6}\right) \\
& =\frac{1}{5}(4)+\frac{4}{5}(4.5)+0(4.7)=\frac{22}{5}=4.4 .
\end{aligned}
$$

Then, we proceed with users that reached node $j$ at instant 4 . This involves loading the flows associated with strategy sets $\bar{S}^{42}, \bar{S}^{43}$ and $\bar{S}^{44} \cup\left\{s^{*}\right\}$. This yields (we skip details) $\pi_{j k}^{s^{*} 4}=0, \pi_{j l}^{s^{*} 4}=7 / 15, \pi_{j}^{s^{*} 4}=8 / 15$ and $\omega_{4 j}^{s^{*} 4}$ and

$$
\begin{aligned}
\omega_{4 j}^{s^{*} 4} & =\pi_{j k}^{s^{*} 4}\left(2+\omega_{6 k}^{s^{*} 6}\right)++\pi_{j l}^{s^{*} 4}\left(2+\omega_{6 l}^{s^{*} 6}\right)+\pi_{j}^{s^{*} 4}\left(1+\omega_{4 j}^{s^{*} 6}\right) \\
& =\frac{7}{15}(4.5)+\frac{8}{15}(5)=\frac{71.5}{15}=4.76 .
\end{aligned}
$$

\section{$5 \quad$ Numerical results}

Numerical results have been obtained using algorithm DSTRATEQ, which mimics the method of successive averages (MSA) for solving monotone variational inequalities. Since the cost mapping $C$ may fail to be monotone, this method is heuristic in our context. At each iteration, we set

$$
x^{k+1}=\left(1-\theta^{k}\right) x^{k}+\theta^{k} \bar{x}\left(x^{k}\right), k=1,2, \ldots
$$

where $\theta^{k} \in(0,1)$ and

$$
\bar{x}_{s}^{t}\left(x^{k}\right)=\left\{\begin{array}{ll}
d_{q r}^{t} & \text { if } s=s^{*} \\
0 & \text { otherwise }
\end{array}, \forall(q, r) \forall s \in W_{q r}^{t}, \forall t \leq T .\right.
$$

For improved convergence, the stepsize was chosen adaptively and independently for each OD pair. More precisely:

$$
\theta_{s}=1-\frac{C^{s^{*}}}{C_{t}^{s}}
$$

The algorithm was halted as soon as the relative gap function became smaller than some predetermined tolerance. We report numerical results on one small and one medium-sized network. 


\subsection{Small network}

Our first test problem is based on the bidirectional network illustrated in Figure 6 . The number of periods $T$ is set to 65 and the latest departure instant $T^{\prime}$ is set to 9 . Additional data for the reference scenario is shown in Tables 2 and 3. A second scenario was constructed by decreasing the capacities of $\operatorname{arcs}(2,3)$ and $(3,2)$ to 5 units, and simultaneously multiplying all demand by the factor $3 / 2$. Key statistics are displayed in Tables 4, 5, 6 and 7, namely: number of active strategies, gap function, CPU time and minimum strategic costs.

In both scenarios, DSTRATEQ converged rapidly to a unique equilibrium solution, from any starting point. The relative gap function decreases to $1 \%$ after roughly 20 iterations for the both networks. Also, as expected, the increase pressure on available capacity in the second scenario yields an increase in the number of active strategies, and to increased CPU time. It can also be readily observed from the output data that FIFO is satisfied, i.e., later departures at the origin node result in later arrivals at the destination. Due to the demand patterns and the system's dynamics, travel delays increase sharply as one approaches the peak period, around instants 6 and 7 .

\subsection{Sioux Falls network}

This classical network (see [13]) illustrated in Figure 7 comprises 24 nodes, 76 arcs and $8 O D$ pairs. The number of periods $T$ and the latest departure time $T^{\prime}$ are set to 100 and 4 , respectively.

Numerical tests have been performed on three scenarios. Tables 8 and 9 contain the relevant input data for the base scenario $\mathbf{A}$. In scenario $\mathbf{B}$, some arc capacities are modified (see Table 10) and demand is multiplied by a factor 2 throughout. The modifications relevant to scenario $\mathbf{C}$ are displayed in Table 11. For each scenario, the control parameters $\epsilon_{1}$ and $\epsilon_{2}$ have been set to 0.1 .

Tables 12, 13 and 14 show that convergence occurs at a reasonable rate, with a relative gap inferior to $1 \%$ reached after less than 20 iterations. As was the case on the small network, the algorithm converged toward a unique equilibrium solution. Tables 15, 16 and 17 give the profiles of minimum travel delays for different departure times. While the nature of the solution changes, both qualitatively and quantitatively, in Scenarios B and $\mathbf{C}$ (note the sharp increase of the size of the working set for Scenario B), the rate of convergence was not greatly influenced by the increased congestion. In particular, the running time of each major iteration remained stable, at around one minute per iteration.

\subsection{Variation of travel delays}

In the strategic model, and equilibrium is reached when average delays are equalized. However, faced with two strategies with equal mean delays, a "risk-averse" user might prefer the one with the smallest standard deviation $\sigma_{s}^{t}$, defined as 


$$
\sigma_{s}^{t}(x)=\left[\sum_{p(t) \in P^{s}} \kappa_{p(t)}^{s}(x)\left(C^{p(t)}\right)^{2}-\left[C_{s}^{t}(x)\right]^{2}\right]^{\frac{1}{2}},
$$

where $\kappa_{p(t)}^{s}(x)$ is the probability of using path $p(t)$ and $C^{p(t)}$ is the actual delay along path $p(t)$.

The inclusion of a variation criterion within the model would make it intractable from a computational point of view. However, we computed, for Sioux Falls' scenario $\mathbf{B}$ the standard deviation of strategic costs for OD pair $(7,20)$, and found a value of 0.5 . This small value is most likely to be perceived as negligible by the users, and validates a posteriori the choice of a single criterion in the equilibrium model.

\section{Conclusion}

In this paper, we have shown that the concept of strategy, previously used in the static transit assignment problem, can be adapted to the realm of dynamic traffic assignment on capacitated networks. This yields a model where the FIFO rule is automatically satisfied. The nonvacuity of the solution set was proved and the implementation showed the applicability of the concept to small networks. Moreover, the numerical results provided interpretable insights on the strategic behavior of users.

This opens up several avenues for further research. One is the extension of the work of [7] on static capacitated transit systems to a dynamic environment. Another topic of research concerns the efficiency of the loading algorithm. While its high running time follows from the number of required elementary operations, which can be evaluated fairly accurately, it is due primarily to the fact that all physical arcs of our test networks are capacitated. Future research will focus on taking advantage of the small number of congested arcs in real networks. Finally, the strategic approach is well suited at addressing situations where random accidents or incidents occur, and where user behaviour is dictated by the appraisal of current traffic conditions, based on past (experience) or present (on-board vehicle) information.

\section{References}

[1] V. Astarita (1996), A continuous time link model for dynamic network loading based on travel time function, Proc. 13th International Symposium on Transportation and Traffic Theory (Tarrytown, N.Y.) (J.B. Lesort, ed.), PergamonElsevier, pp. 79-102.

[2] M. Carey (1992), Nonconvexity of the dynamic traffic assignment problem, Transportation Research B26, pp. 127-133.

[3] O. Drissi-Kaitouni, A. Hamada-Benchekroun (1992), A dynamic traffic assignment model and a solution algorithm, Transportation Science 26, pp. 119-128. 
[4] O. Drissi-Kaitouni, M. Gendreau (1992), A new dynamic traffic assignment model, Publication CRT-854, Centre de recherche sur les transports, Université de Montréal.

[5] T.L. Friesz, D. Bernstein, T.E. Smith, R. Tobin et B.W Wie (1993), A variational inequality formulation on the dynamic network user equilibrium problem, Operations Research 41, pp. 179-191.

[6] Y. Hamdouch (2002), Affectation statique et dynamique des usagers dans un réseau de transport avec capacités rigides, Ph.D dissertation, Département d'informatique et de recherche opérationnelle, Université de Montréal.

[7] Y. Hamdouch, P. Marcotte and S. Nguyen (2002), Capacitated traffic assignment with priority rules, preprint.

[8] P. Marcotte and S. Nguyen (1998), Hyperpath formulations of traffic assignment problems, Equilibrium and Advanced Transportation Modelling (P. Marcotte and S. Nguyen, eds.), Kluwer Academic Publisher, pp. 175-199.

[9] P. Marcotte, S. Nguyen and A. Schoeb (2000), A strategic flow model of traffic assignment in capacitated networks, Publication 2000-10, Centre de recherche sur les transports, Université de Montréal.

[10] D. Merchant et G. Nemhauser (1978), A model and an algorithm for the dynamic traffic assignment problems, Transportation Science 12, pp. 183-199.

[11] S. Nguyen and S. Pallottino (1989), Hyperpaths and shortest hyperpaths, In B. Simeone, editor, Combinatorial Optimization, volume 1403 of Lecture Notes in Mathematics, pp. 258-271, Springer-Verlag, Berlin.

[12] S. Nguyen, S. Pallottino et P. Malucelli (2001), A modeling framework for the passenger assignment on a transport network with time-tables, Transportation Science 55B, pp. 238-249.

[13] C. Suwansirikul, T.L. Friesz and R.L. Tobin (1987), Equilibrium decomposition optimization: a heuristic for the continuous equilibrium network design problem, Transportation science 21, pp. 254-263.

[14] J.G. Wardrop (1952), Some theoretical aspects of road traffic research, Proceedings of the Institution of Civil Engineers, Part II, pp. 325-378.

[15] D. Zawack et G. Thompson (1987), A dynamic space-time network flow model for city traffic congestion, Transportation Science 21, pp. 153-162. 


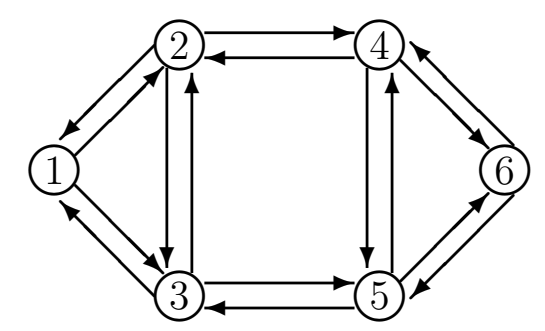

Figure 6: Small network $(T=65)$

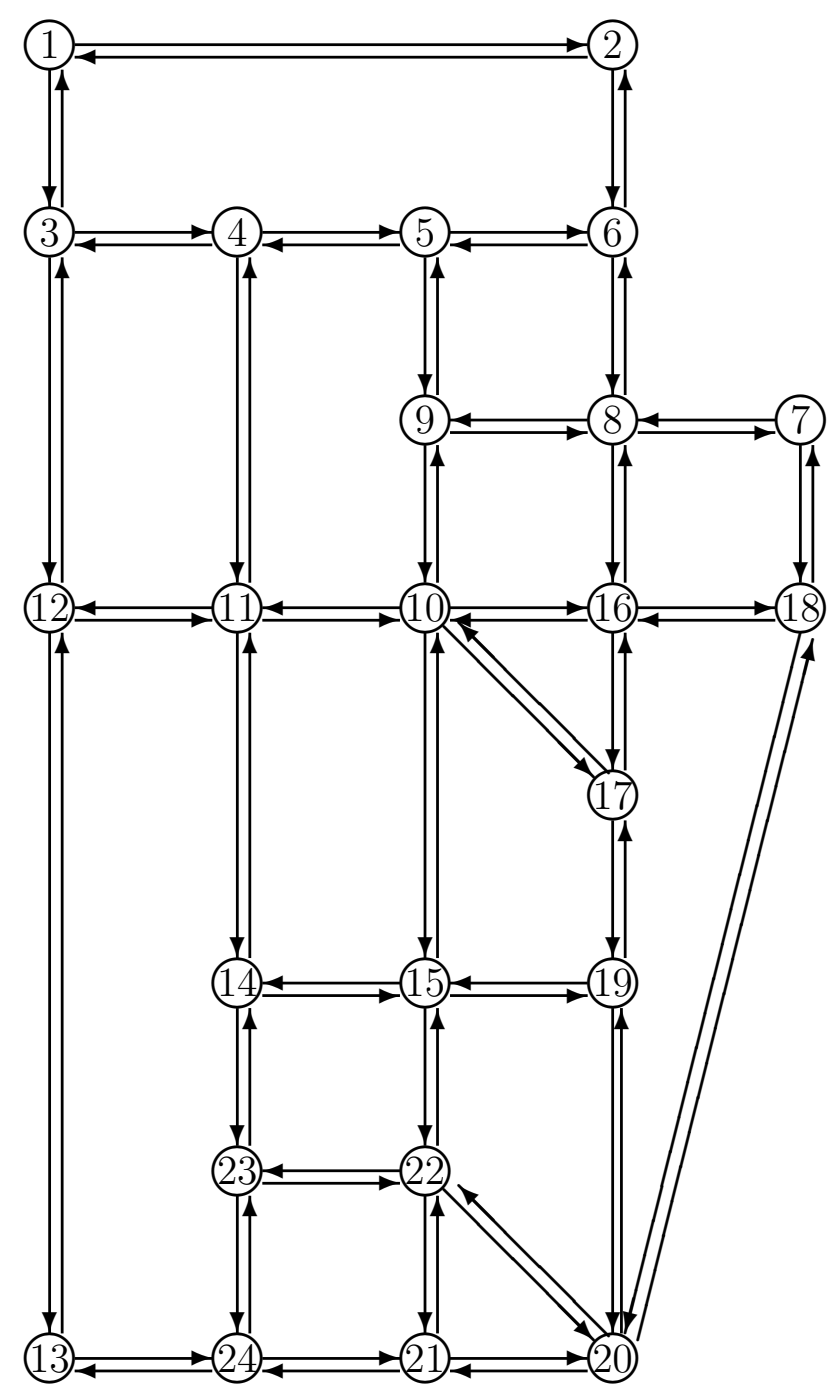

Figure 7: Sioux Falls network 


\begin{tabular}{ccc|ccc} 
Arc & Cost & Capacity & Arc & Cost & Capacity \\
\hline$(1,2)$ & 3 & 20 & $(3,5)$ & 4 & 10 \\
$(2,1)$ & 3 & 20 & $(5,3)$ & 4 & 10 \\
$(1,3)$ & 2 & 20 & $(4,5)$ & 4 & 10 \\
$(3,1)$ & 2 & 20 & $(5,4)$ & 4 & 10 \\
$(2,3)$ & 1 & 10 & $(4,6)$ & 5 & 25 \\
$(3,2)$ & 1 & 10 & $(6,4)$ & 5 & 25 \\
$(2,4)$ & 2 & 10 & $(5,6)$ & 6 & 25 \\
$(4,2)$ & 2 & 10 & $(6,5)$ & 6 & 25
\end{tabular}

Table 2: Small network: arc data

\begin{tabular}{cc|cc}
$O D(t)$ & Demand & $O D(t)$ & Demand \\
\hline$(1,6,0)$ & 5 & $(6,1,0)$ & 10 \\
$(1,6,1)$ & 10 & $(6,1,1)$ & 15 \\
$(1,6,2)$ & 25 & $(6,1,2)$ & 30 \\
$(1,6,3)$ & 18 & $(6,1,3)$ & 23 \\
$(1,6,4)$ & 15 & $(6,1,4)$ & 20 \\
$(1,6,5)$ & 17 & $(6,1,5)$ & 22 \\
$(1,6,6)$ & 30 & $(6,1,6)$ & 35 \\
$(1,6,7)$ & 45 & $(6,1,7)$ & 50 \\
$(1,6,8)$ & 15 & $(6,1,8)$ & 20 \\
$(1,6,9)$ & 12 & $(6,1,9)$ & 17
\end{tabular}

Table 3: Small network: OD information 


\begin{tabular}{rrrr} 
\# iter. & $|W|$ & Gap (\%) & Cpu (sec) \\
\hline 0 & 20 & 26.4923 & 3.52 \\
1 & 34 & 11.3380 & 8.47 \\
2 & 34 & 5.5682 & 13.37 \\
5 & 36 & 2.8952 & 28.36 \\
10 & 40 & 1.5934 & 54.81 \\
20 & 40 & 0.8830 & 111.27 \\
30 & 40 & 0.5842 & 164.44 \\
50 & 40 & 0.3081 & 275.80 \\
100 & 40 & 0.0817 & 555.09
\end{tabular}

Table 4: Small network: results of first scenario

\begin{tabular}{lc|cc}
$O D(t)$ & Minimum cost & $O D(t)$ & Minimum cost \\
\hline$(1,6,0)$ & 10.000 & $(6,1,0)$ & 10.000 \\
$(1,6,1)$ & 10.000 & $(6,1,1)$ & 10.333 \\
$(1,6,2)$ & 10.799 & $(6,1,2)$ & 11.333 \\
$(1,6,3)$ & 11.888 & $(6,1,3)$ & 12.156 \\
$(1,6,4)$ & 12.000 & $(6,1,4)$ & 12.189 \\
$(1,6,5)$ & 12.000 & $(6,1,5)$ & 12.263 \\
$(1,6,6)$ & 12.209 & $(6,1,6)$ & 12.617 \\
$(1,6,7)$ & 13.075 & $(6,1,7)$ & 13.831 \\
$(1,6,8)$ & 13.360 & $(6,1,8)$ & 14.536 \\
$(1,6,9)$ & 13.018 & $(6,1,9)$ & 14.555
\end{tabular}

Table 5: Small network: minimum costs after 100 iterations (first scenario) 


\begin{tabular}{rrrr} 
\# iter. & $|W|$ & Gap (\%) & $\mathrm{Cpu}(\mathrm{sec})$ \\
\hline 0 & 20 & 40.8394 & 4.12 \\
1 & 36 & 13.3624 & 9.61 \\
2 & 36 & 6.1367 & 15.00 \\
5 & 40 & 3.8735 & 32.16 \\
10 & 47 & 2.1099 & 64.32 \\
20 & 53 & 1.0616 & 135.83 \\
30 & 55 & 0.7803 & 210.38 \\
50 & 57 & 0.4901 & 367.39 \\
100 & 57 & 0.2222 & 765.75
\end{tabular}

Table 6: Small network: results of second scenario

\begin{tabular}{lccc}
$O D(t)$ & Minimum cost & $O D(t)$ & Minimum cost \\
\hline$(1,6,0)$ & 10.000 & $(6,1,0)$ & 10.333 \\
$(1,6,1)$ & 10.333 & $(6,1,1)$ & 11.111 \\
$(1,6,2)$ & 11.528 & $(6,1,2)$ & 12.499 \\
$(1,6,3)$ & 12.347 & $(6,1,3)$ & 13.492 \\
$(1,6,4)$ & 12.508 & $(6,1,4)$ & 14.133 \\
$(1,6,5)$ & 12.674 & $(6,1,5)$ & 14.641 \\
$(1,6,6)$ & 13.501 & $(6,1,6)$ & 15.808 \\
$(1,6,7)$ & 15.289 & $(6,1,7)$ & 18.134 \\
$(1,6,8)$ & 16.613 & $(6,1,8)$ & 19.669 \\
$(1,6,9)$ & 16.188 & $(6,1,9)$ & 19.992
\end{tabular}

Table 7: Small network: minimum costs after 100 iterations (second scenario) 


\begin{tabular}{crc|ccc} 
Arc & Cost & Capacity & Arc & Cost & Capacity \\
\hline & & & & & \\
$(1,2)$ & 6 & 60 & $(13,24)$ & 4 & 60 \\
$(1,3)$ & 2 & 60 & $(14,11)$ & 3 & 15 \\
$(2,1)$ & 6 & 60 & $(14,15)$ & 4 & 60 \\
$(2,6)$ & 2 & 60 & $(14,23)$ & 2 & 10 \\
$(3,1)$ & 2 & 60 & $(15,10)$ & 4 & 20 \\
$(3,4)$ & 2 & 60 & $(15,14)$ & 4 & 60 \\
$(3,12)$ & 4 & 60 & $(15,19)$ & 2 & 60 \\
$(4,3)$ & 2 & 60 & $(15,22)$ & 2 & 60 \\
$(4,5)$ & 2 & 60 & $(16,8)$ & 2 & 40 \\
$(4,11)$ & 3 & 10 & $(16,10)$ & 2 & 60 \\
$(5,4)$ & 2 & 60 & $(16,17)$ & 2 & 40 \\
$(5,6)$ & 10 & 60 & $(16,18)$ & 2 & 60 \\
$(5,9)$ & 2 & 20 & $(17,10)$ & 6 & 60 \\
$(6,2)$ & 2 & 60 & $(17,16)$ & 2 & 40 \\
$(6,5)$ & 10 & 60 & $(17,19)$ & 6 & 60 \\
$(6,8)$ & 2 & 60 & $(18,7)$ & 4 & 60 \\
$(7,8)$ & 2 & 60 & $(18,16)$ & 2 & 60 \\
$(7,18)$ & 4 & 60 & $(18,20)$ & 16 & 60 \\
$(8,6)$ & 2 & 60 & $(19,15)$ & 2 & 60 \\
$(8,7)$ & 2 & 60 & $(19,17)$ & 6 & 60 \\
$(8,9)$ & 4 & 60 & $(19,20)$ & 4 & 30 \\
$(8,16)$ & 2 & 40 & $(20,18)$ & 16 & 60 \\
$(9,5)$ & 2 & 20 & $(20,19)$ & 4 & 30 \\
$(9,8)$ & 4 & 60 & $(20,21)$ & 4 & 60 \\
$(9,10)$ & 2 & 20 & $(20,22)$ & 6 & 10 \\
$(10,9)$ & 2 & 20 & $(21,20)$ & 4 & 60 \\
$(10,11)$ & 3 & 10 & $(21,22)$ & 8 & 60 \\
$(10,15)$ & 4 & 20 & $(21,24)$ & 4 & 60 \\
$(10,16)$ & 2 & 60 & $(22,15)$ & 2 & 60 \\
$(10,17)$ & 6 & 60 & $(22,20)$ & 6 & 10 \\
$(11,4)$ & 3 & 10 & $(22,21)$ & 8 & 60 \\
$(11,10)$ & 3 & 10 & $(22,23)$ & 2 & 10 \\
$(11,12)$ & 4 & 60 & $(23,14)$ & 2 & 10 \\
$(11,14)$ & 3 & 15 & $(23,22)$ & 2 & 10 \\
$(12,3)$ & 4 & 60 & $(23,24)$ & 2 & 60 \\
$(12,11)$ & 4 & 60 & $(24,13)$ & 4 & 60 \\
$(12,13)$ & 14 & 60 & $(24,21)$ & 4 & 60 \\
$(13,12)$ & 14 & 60 & $(24,23)$ & 2 & 60 \\
& & & & &
\end{tabular}

Table 8: Sioux Falls: arc data 


\begin{tabular}{|c|c|c|c|}
\hline$O D(t)$ & Demand & $O D(t)$ & Demand \\
\hline$(1,20,0)$ & 25 & $(20,1,0)$ & 25 \\
\hline$(1,20,1)$ & 40 & $(20,1,1)$ & 40 \\
\hline$(1,20,2)$ & 55 & $(20,1,2)$ & 55 \\
\hline$(1,20,3)$ & 30 & $(20,1,3)$ & 30 \\
\hline$(1,20,4)$ & 20 & $(20,1,4)$ & 20 \\
\hline$(1,24,0)$ & 20 & $(24,1,0)$ & 20 \\
\hline$(1,24,1)$ & 35 & $(24,1,1)$ & 35 \\
\hline$(1,24,2)$ & 50 & $(24,1,2)$ & 50 \\
\hline$(1,24,3)$ & 25 & $(24,1,3)$ & 25 \\
\hline$(1,24,4)$ & 15 & $(24,1,4)$ & 15 \\
\hline$(7,20,0)$ & 15 & $(20,7,0)$ & 15 \\
\hline$(7,20,1)$ & 30 & $(20,7,1)$ & 30 \\
\hline$(7,20,2)$ & 45 & $(20,7,2)$ & 45 \\
\hline$(7,20,3)$ & 20 & $(20,7,3)$ & 20 \\
\hline$(7,20,4)$ & 10 & $(20,7,4)$ & 10 \\
\hline$(7,24,0)$ & 15 & $(24,7,0)$ & 15 \\
\hline$(7,24,1)$ & 30 & $(24,7,1)$ & 30 \\
\hline$(7,24,2)$ & 45 & $(24,7,2)$ & 45 \\
\hline$(7,24,3)$ & 20 & $(24,7,3)$ & 20 \\
\hline$(7,24,4)$ & 10 & $(24,7,4)$ & 10 \\
\hline
\end{tabular}

Table 9: Sioux Falls: OD information (scenario A)

\begin{tabular}{cc|cc} 
Arc & Capacity & Arc & Capacity \\
\hline$(8,16)$ & 40 & $(16,8)$ & 40 \\
$(16,17)$ & 40 & $(17,16)$ & 40 \\
$(19,20)$ & 30 & $(20,19)$ & 30
\end{tabular}

Table 10: Sioux Falls: arc capacities for scenario B 


\begin{tabular}{cr} 
Pair $O D(t)$ & Demand \\
\hline$(7,20,0)$ & 60 \\
$(7,20,1)$ & 120 \\
$(7,20,2)$ & 180 \\
$(7,20,3)$ & 80 \\
$(7,20,4)$ & 40
\end{tabular}

Table 11: Sioux Falls: demand for OD pair $(7,20)$ (scenario C)

\begin{tabular}{rrrr} 
\# iter. & $|W|$ & Gap (\%) & Cpu (sec) \\
\hline 0 & 40 & 6.3853 & 49.81 \\
1 & 64 & 5.7933 & 118.56 \\
2 & 67 & 4.9266 & 188.85 \\
5 & 70 & 2.9340 & 406.69 \\
10 & 77 & 1.5637 & 788.17 \\
20 & 78 & 0.7630 & 1595.60 \\
30 & 79 & 0.4816 & 2426.13 \\
50 & 80 & 0.3279 & 4157.09
\end{tabular}

Table 12: Sioux Falls results (scenario A)

\begin{tabular}{rrrr} 
\# iter. & $|W|$ & Gap (\%) & Cpu (sec) \\
\hline 0 & 40 & 32.5518 & 55.39 \\
1 & 75 & 20.2456 & 139.39 \\
2 & 92 & 14.4513 & 236.32 \\
5 & 123 & 7.0109 & 577.66 \\
10 & 149 & 3.5233 & 1250.41 \\
20 & 168 & 1.4144 & 2811.61 \\
30 & 171 & 0.9505 & 4479.48 \\
50 & 172 & 0.5053 & 7894.81
\end{tabular}

Table 13: Sioux Falls results (scenario B) 


\begin{tabular}{rrrr} 
\# iter. & $|W|$ & Gap (\%) & Cpu (sec) \\
\hline 0 & 40 & 38.6861 & 56.49 \\
1 & 76 & 24.6751 & 142.80 \\
2 & 93 & 16.0157 & 240.55 \\
5 & 130 & 7.5075 & 593.06 \\
10 & 159 & 3.6671 & 1301.51 \\
20 & 186 & 1.5922 & 2970.06 \\
30 & 193 & 0.9470 & 4763.01 \\
50 & 192 & 0.6316 & 8447.17
\end{tabular}

Table 14: Sioux Falls results (scenario C) 


\begin{tabular}{lc|cc}
$O D(t)$ & Minimum cost & $O D(t)$ & Minimum cost \\
\hline$(1,20,0)$ & 20.692 & $(20,1,0)$ & 20.253 \\
$(1,20,1)$ & 21.563 & $(20,1,1)$ & 21.009 \\
$(1,20,2)$ & 22.013 & $(20,1,2)$ & 22.646 \\
$(1,20,3)$ & 23.342 & $(20,1,3)$ & 23.270 \\
$(1,20,4)$ & 23.401 & $(20,1,4)$ & 23.374 \\
\hline & & $(24,1,0)$ & 14.956 \\
$(1,24,0)$ & 14.914 & $(24,1,1)$ & 17.157 \\
$(1,24,1)$ & 17.550 & $(24,1,2)$ & 20.839 \\
$(1,24,2)$ & 20.783 & $(24,1,3)$ & 23.031 \\
$(1,24,3)$ & 23.439 & $(24,1,4)$ & 23.487 \\
$(1,24,4)$ & 23.419 & $(24,0)$ & 16.087 \\
\hline$(7,20,0)$ & 16.000 & $(20,7,0)$ & 16.250 \\
$(7,20,1)$ & 16.333 & $(20,7,1)$ & 16.997 \\
$(7,20,2)$ & 17.212 & $(20,7,2)$ & 18.559 \\
$(7,20,3)$ & 17.461 & $(20,7,3)$ & 19.239 \\
$(7,20,4)$ & 17.447 & $(20,7,4)$ & 19.373 \\
\hline$(7,24,0)$ & 16.087 & $(24,4)$ & 21.549 \\
$(7,24,1)$ & 16.910 & $(24,3)$ & 19.683 \\
$(7,24,2)$ & 19.283 & & \\
$(7,24,3)$ & 20.659 & $(21.546$ \\
$(7,24,4)$ & 21.416 & & \\
& & & \\
\hline
\end{tabular}

Table 15: Sioux Falls: minimum costs after 50 iterations (scenario A) 


\begin{tabular}{|c|c|c|c|}
\hline$O D(t)$ & Minimum cost & $O D(t)$ & Minimum cost \\
\hline$(1,20,0)$ & 24.806 & $(20,1,0)$ & 22.078 \\
\hline$(1,20,1)$ & 27.135 & $(20,1,1)$ & 25.708 \\
\hline$(1,20,2)$ & 29.592 & $(20,1,2)$ & 29.207 \\
\hline$(1,20,3)$ & 31.631 & $(20,1,3)$ & 30.134 \\
\hline$(1,20,4)$ & 31.910 & $(20,1,4)$ & 30.124 \\
\hline$(1,24,0)$ & 18.752 & $(24,1,0)$ & 16.348 \\
\hline$(1,24,1)$ & 23.608 & $(24,1,1)$ & 21.325 \\
\hline$(1,24,2)$ & 25.624 & $(24,1,2)$ & 24.833 \\
\hline$(1,24,3)$ & 26.465 & $(24,1,3)$ & 25.430 \\
\hline$(1,24,4)$ & 26.589 & $(24,1,4)$ & 25.009 \\
\hline$(7,20,0)$ & 16.750 & $(20,7,0)$ & 17.920 \\
\hline$(7,20,1)$ & 19.263 & $(20,7,1)$ & 19.892 \\
\hline$(7,20,2)$ & 20.586 & $(20,7,2)$ & 20.350 \\
\hline$(7,20,3)$ & 20.989 & $(20,7,3)$ & 20.709 \\
\hline$(7,20,4)$ & 20.114 & $(20,7,4)$ & 20.467 \\
\hline$(7,24,0)$ & 16.882 & $(24,0)$ & 16.873 \\
\hline$(7,24,1)$ & 20.448 & $(24,1)$ & 20.944 \\
\hline$(7,24,2)$ & 26.144 & $(24,2)$ & 26.343 \\
\hline$(7,24,3)$ & 28.737 & $(24,3)$ & 27.814 \\
\hline$(7,24,4)$ & 28.108 & $(24,4)$ & 26.843 \\
\hline
\end{tabular}

Table 16: Sioux Falls: minimum costs after 50 iterations (scenario B) 


\begin{tabular}{|c|c|c|c|}
\hline$O D(t)$ & Minimum cost & $O D(t)$ & Minimum costs \\
\hline$(1,20,0)$ & 25.357 & $(20,1,0)$ & 22.078 \\
\hline$(1,20,1)$ & 27.843 & $(20,1,1)$ & 25.714 \\
\hline$(1,20,2)$ & 30.383 & $(20,1,2)$ & 29.081 \\
\hline$(1,20,3)$ & 32.000 & $(20,1,3)$ & 30.228 \\
\hline$(1,20,4)$ & 31.835 & $(20,1,4)$ & 30.204 \\
\hline$(1,24,0)$ & 18.600 & $(24,1,0)$ & 16.348 \\
\hline$(1,24,1)$ & 23.785 & $(24,1,1)$ & 21.323 \\
\hline$(1,24,2)$ & 25.707 & $(24,1,2)$ & 24.835 \\
\hline$(1,24,3)$ & 26.205 & $(24,1,3)$ & 25.437 \\
\hline$(1,24,4)$ & 26.224 & $(24,1,4)$ & 25.019 \\
\hline$(7,20,0)$ & 17.686 & $(20,7,0)$ & 17.920 \\
\hline$(7,20,1)$ & 20.312 & $(20,7,1)$ & 19.892 \\
\hline$(7,20,2)$ & 21.872 & $(20,7,2)$ & 20.363 \\
\hline$(7,20,3)$ & 22.567 & $(20,7,3)$ & 20.681 \\
\hline$(7,20,4)$ & 22.138 & $(20,7,4)$ & 20.462 \\
\hline$(7,24,0)$ & 17.461 & $(24,0)$ & 16.873 \\
\hline$(7,24,1)$ & 21.144 & $(24,1)$ & 20.940 \\
\hline$(7,24,2)$ & 26.823 & $(24,2)$ & 26.318 \\
\hline$(7,24,3)$ & 29.808 & $(24,3)$ & 27.819 \\
\hline$(7,24,4)$ & 29.658 & $(24,4)$ & 26.880 \\
\hline
\end{tabular}

Table 17: Sioux Falls: minimum costs after 50 iterations (scenario C) 


\section{APPENDIX}

\section{ALGORITHM DCAPLOAD $(x)$}

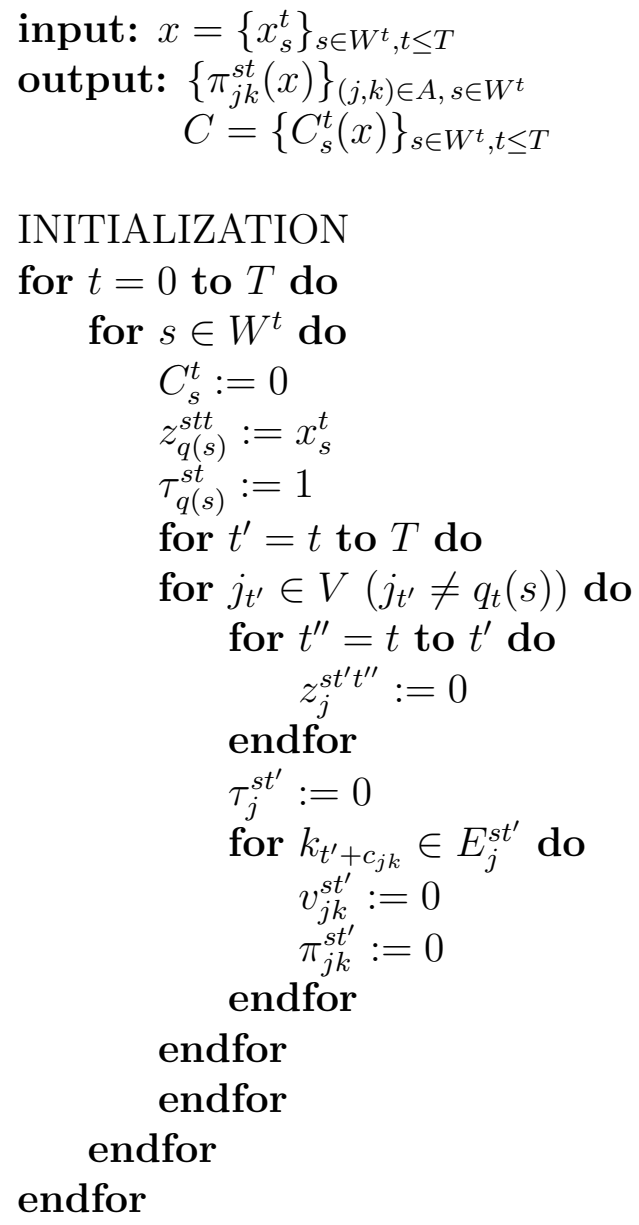

\section{LOADING PHASE}

for $t=0$ to $T$ do

for $j_{t} \in V$ do

for $k_{t+c_{j k}} \in j_{t}^{+}$do

$\bar{u}_{j k}:=u_{j k}$

endfor

$$
\bar{u}_{j}:=\infty
$$

for $t^{\prime}=0$ to $t$ do

$$
\begin{aligned}
& \quad z_{j}^{s t}:=z_{j}^{s t}+z_{j}^{s t t^{\prime}} \\
& \qquad \bar{S}^{t t^{\prime}}:=\left\{s \in W: z_{j}^{s t t^{\prime}}>0\right\} \\
& \text { endfor } \\
& \text { for } t^{\prime}=0 \text { to } t \text { do } \\
& \text { for } s \in \bar{S}^{t t^{\prime}} \text { do }
\end{aligned}
$$$$
\text { endfor }
$$

[strategic volume vector] [conditional arc probabilities] [vector of strategic costs]

[cost of strategy $s$ ] [volume at origine node $q_{t}(s)$ ] [probability of accessing $\left.q_{t}(s)\right]$

[strategic volume at node $j_{t^{\prime}}$ if $j$ has been reached at instant $\left.t^{\prime \prime}\right]$ [conditional probability of accessing node $j_{t^{\prime}}$ using strategy $\left.s\right]$ [strategic volume on arc $\left(j_{t^{\prime}}, k_{t^{\prime}+c_{j k}}\right)$ ] [conditional probability of accessing $\operatorname{arc}\left(j_{t^{\prime}}, k_{t^{\prime}+c_{j k}}\right)$ using strategy $\left.s\right]$ [time topological order]

[residual capacity of $\left.\operatorname{arc}\left(j_{t}, k_{t+c_{j k}}\right)\right]$

$\left[\left(j_{t}, j_{t+1}\right)\right.$ is uncapacitated $]$

[total strategic volume $z_{j}^{s t}$ ]

[set of active strategies at $j_{t}$ according to instant of accessing node $j]$ 


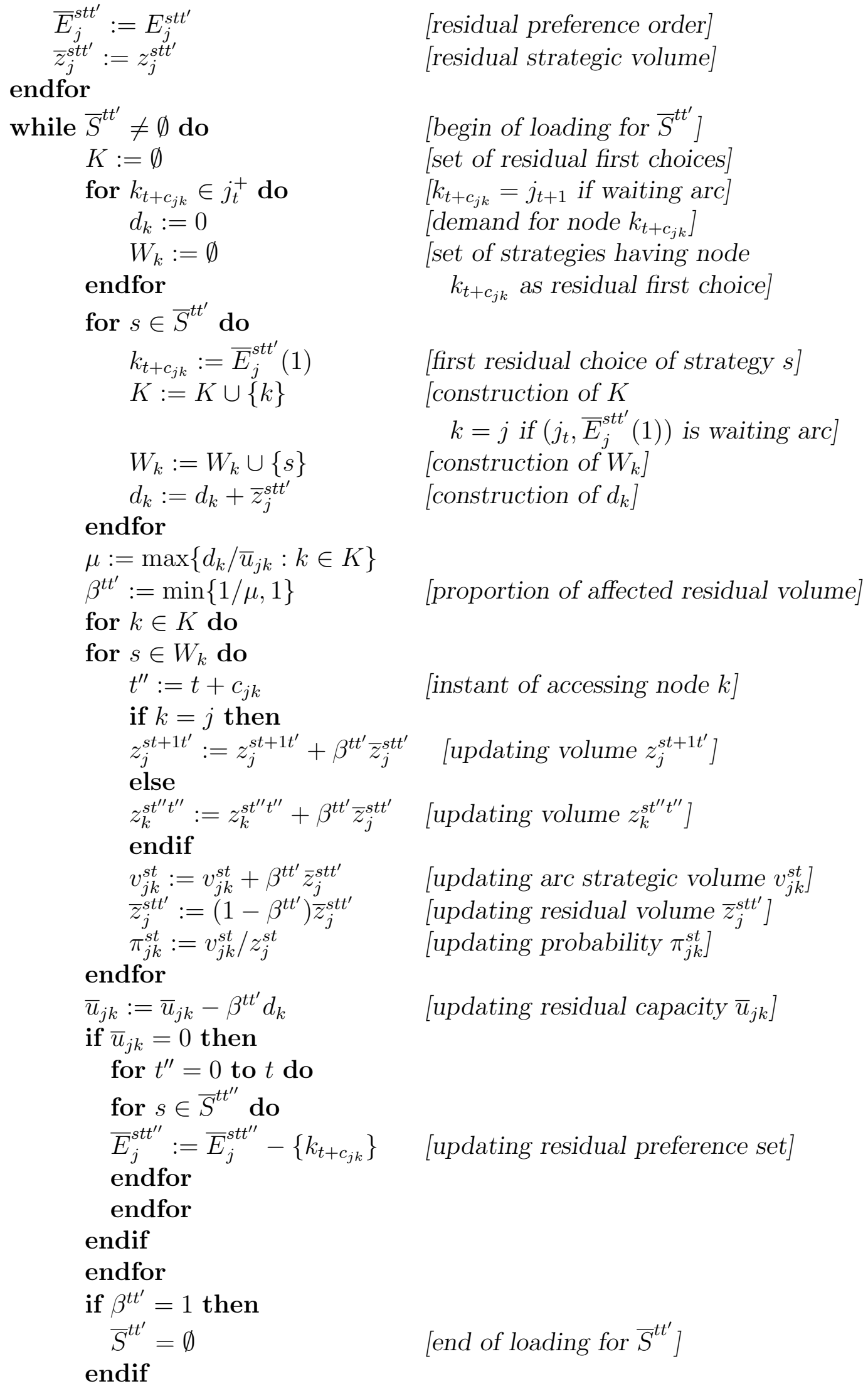


endfor

endfor

for $s \in W^{t}$

for $t^{\prime}=t$ to $T$

for $j_{t^{\prime}} \in V$

for $k_{t^{\prime}+c_{j k}} \in j_{t^{\prime}}^{+}$do

$t^{\prime \prime}=t^{\prime}+c_{j k}$

$C_{s}^{t}:=C_{s}^{t}+c_{j k} \tau_{j}^{s t^{\prime}} \pi_{j k}^{s t^{\prime}}$

$\tau_{k}^{s t^{\prime \prime}}:=\tau_{k}^{s t^{\prime \prime}}+\tau_{j}^{s t^{\prime}} \pi_{j k}^{s t^{\prime}}$

endfor

$C_{s}^{t}:=C_{s}^{t}+\tau_{j}^{s t^{\prime}} \pi_{j}^{s t^{\prime}}$

endfor

$\tau_{j}^{s\left(t^{\prime}+1\right)}:=\tau_{j}^{s\left(t^{\prime}+1\right)}+\tau_{j}^{s t^{\prime}} \pi_{j}^{s t^{\prime}}$ [period of accessing node $k$ ]

[update of cost $C_{s}^{t}$ ]

[update of probability $\tau_{k}^{s t^{\prime \prime}}$ ]

[update of cost $C_{s}^{t}$ ]

[update of probability $\tau_{j}^{s\left(t^{\prime}+1\right)}$ ]

endfor

endfor

endfor 